\title{
MRI Compatible Robot Systems for Medical Intervention
}

\author{
Ming Li, Dumitru Mazilu, Ankur Kapoor \\ and Keith A. Horvath \\ National Institutes of Health \\ USA
}

\section{Introduction}

Magnetic resonance imaging (MRI) is a non-invasive medical imaging tool that helps physicians diagnose and treat medical conditions. It offers excellent visualization of softtissue in any imaging plane without using of iodinated contrast agent or ionizing radiation. In addition to anatomical morphology, MRI can also provide functional information.

With novel fast imaging technologies, MRI became an imaging tool for guiding and monitoring various interventions and biopsy procedures on various organs including the brain, breast, prostate, liver and spine (Jolesz, 1998, Melzer \& Seibel, 1999). High performance magnetic field gradients, multi-channel receivers, and advanced reconstruction systems improve the clinical applicability of real time MRI procedures (Nayak et al., 2004, Bock et al., 2004, Guttman et al., 2003, Lederman, 2005). Modern scanners designed for the interventional environment can provide real-time images of acceptable quality in excess of 10 frames per second. As a result, real-time MRI (rtMRI) is becoming an attractive method for many minimally invasive cardiac interventions (Kuehne et al., 2003, Raval et al., 2006, Henk et al., 2005, McVeigh et al., 2006, Horvath et al., 2007).

However the confined physical space of MRI scanner challenges medical intervention, even the magnets with open architecture provide only a limited working space, at the expense of image quality. The use of robots inside the MRI scanner is a very attractive solution: a robot manipulates the intervention instruments while MR images continuously give feedback of the position of the instruments which are controlled by the robot. MR compatible robotic systems have been researched and developed for prostate biopsy and brachytherapy (Chinzei et al., 2000, Krieger et al., 2005, Fischer et al., 2006, Stoianovici et al., 2007a), breast intervention (Kaiser et al., 2000, Larson et al., 2004), interventional spinal procedure (Hempel et al., 2003), neurosurgery (Masamune et al., 1995, Koseki et al., 2002), interventional liver therapy (Hata et al., 2005, Kim et al., 2002), and cardiac intervention (Li et al., 2008).

Design of a system operating inside or close to the bore of a high field MRI scanner is of significant complexity. Due to the strong magnetic field of the MRI, standard materials, sensors and actuators cannot be employed. Due to the confined space of MRI bore, the mechanical design should be compact and simultaneously functional. In this chapter, we 
discuss MRI robots for medical interventions, including the MR compatibility, mechanical design and control method. As an example, we present our work on the development of a robotic valve delivery module for transapical aortic valve replacement under real-time MRI guidance. Furthermore, we present our work on an MR compatible hands-on cooperative control of a pneumatically actuated robot.

\section{MR compatibility}

Three basic features for high quality MRI are high and homogenous magnetic fields, fastswitching magnetic field gradients and radiofrequency (RF) pulses. These extreme environmental conditions require adequate construction materials, actuation assemblies, sensors, and proper shielding of electronics. For example, in strong magnetic fields, ferromagnetic materials can be dangerous projectiles. Also, the homogeneity of the main magnetic field is strongly affected by ferromagnetic materials inside the MRI scanner, resulting in substantial distortion of images. Fast changing gradient magnetic fields can induce electrical fields and eddy-currents inside conductive materials. These eddy-currents can heat conductive materials and distort the homogeneity of the main magnetic field and thus severely affect the quality of MR images. RF pulses can heat conductive elements, such as wires or interventional devices. Devices containing closed metallic loops should be avoided in the MR environment.

MRI is also very sensitive to electromagnetic noise. Electronic equipment and wires needed for the operation of mechatronic devices placed inside the MRI scanner room can generate electromagnetic noise if these are not properly shielded.

MRI compatibility of robots has different levels: 1) MR safety, no risk to the patient or medical personnel; 2) image quality not affected by the introduction and operation of the robot; and 3) the operation of the robot not affected by the magnetic fields (Schenck, 1998, Schenck, 2000, Chinzei, 1999). We present MR compatibility of different materials, actuators and sensors and discuss the level of the compatibility of each of these in this section.

\subsection{Materials}

Based on their reaction in a magnetic field, the materials can be characterized in to three categories: ferromagnetic, paramagnetic and diamagnetic materials. Ferromagnetic materials, such as iron, magnetic stainless steel, cobalt and nickel, exhibit a strong attraction to a magnetic field. These materials are not MR safe. They could be a dangerous projectile to patients, physicians, and the MRI scanner when they are close to the scanner. Moreover, ferromagnetic materials create positive susceptibility to an external magnetic field. The homogeneity of an MR static field will be disturbed by the presence of ferromagnetic materials; therefore the image will be distorted. Yet, the ferromagnetic materials, such as magnetic stainless steel, have desirable mechanical properties. Paramagnetic materials, such as aluminium, platinum and austenitic stainless steels, become slightly magnetized when exposed to a magnetic field. Paramagnetic metals have a small and positive susceptibility to magnetic fields; hence they require some compromise when they are used inside an MRI scanner and close to imaging areas. Diamagnetic metals have a very weak and negative susceptibility to magnetic fields. Copper, silver, and gold are diamagnetic. They are MR safe, and will not affect the homogeneity of a static magnetic field when they are placed 
close to an MRI scanner. They have very limited or negligible image artifacts even they are located close to imaging areas.

Another effect of MR compatibility is the generation of the eddy-currents inside conductive materials due to fast-switching gradient magnetic fields. These eddy-currents may alter the local homogeneity of the main magnetic field and affect the quality and linearity of MR images, causing image artifacts. Moreover, eddy-currents may also cause unwanted heating of the materials.

Materials suitable for MR-compatible devices in level 3 are nonmagnetic and nonconductive. Plastics, ceramics and composite material have been used in the development of MR-compatible systems. But plastics are often too soft and present limited structural stiffness, whereas, ceramics are too hard, brittle and could lead to manufacturing difficulties or part failures. Bearings, ball screws and gears made of non-ferromagnetic metals, such as aluminium, copper, brass, titanium, and tantalum are often used in MR compatible systems. These metal parts do not present substantial problems or image artifacts as long as they are of small size and appropriately positioned relative to the imaging areas.

In conclusion, MR-compatible materials can be characterized into three categories depending on their magnetic compatibility when they are placed inside an MRI scanner:

1) materials that produce no image distortion: nylon, vespel, PEEK (poly-ether-etherketone), polysulfone, zirconia, plexiglass. Polyimide (from class of vespel materials) for example, presents outstanding structural strength, good chemical resistance, very low electrical and thermal conductivity, low friction and have no detectable MR artifacts.

2) materials that produce low level of image distortion, but for many applications are acceptable: brass, zinc, lead.

3) materials that produce noticeable image distortion, but for some application are still acceptable: titanium, tantalum, tungsten, zirconium, molybdenum, aluminium.

The MR compatibility of certain types of materials and devices have been reported in (Chinzei et al., 1999, Schenck, 2000). In order to be suitable for medical devices or medical robotics, these materials also should be biocompatible, sterilizable and economical.

\subsection{Actuators}

Electromagnetic motors are commonly used to actuate conventional robotic system, yet they are not safe and compatible in an MRI environment. The principle of electromagnetic motor operation is that a mechanical force is produced by the interaction of an electric current and a magnetic field. Electromagnetic motors usually are equipped with magnets to maintain a magnetic field. When such an electromagnetic motor is placed close to MRI bore, it will obviously disturb the homogeneity of the MR static field. On the other hand, the static magnetic field and the fast-switching gradient magnetic field will also affect the operation of the motor.

Ultrasonic or piezoelectric motors are used in various MRI compatible robotic systems (Masamune et al., 1995, Chinzei et al., 2000, Kaiser et al., 2000, Koseki et al., 2002, Elhawary et al., 2007). These motors are driven by the ultrasonic vibration of a piezoelectric transducer when high-frequency voltage is applied; therefore theoretically they do not produce magnetic fields and are immune to the magnetic field. According to (Chinzei et al., 1999 and Fischer et al., 2008a), some commercially available ultrasonic motors are prone to cause image artifacts and significant signal-to-noise ratio (SNR) loss. The advantages of ultrasonic 
motors include compact shape, small size, bidirectional and high torque output. The high breaking torque allows the robot to maintain its position even when it is not be powered. But these motors are not back-drivable, mechanical clutches need to be implemented to manually move the robot without detaching the mechanical part and the motor in the case of a medical emergency. For interventions which require simultaneous use of the imager and the robot, the ultrasonic motors should be placed outside of the MRI scanner. A motion transmission system, such as shafts, belts/chains, cables and linkages, is required to transfer the motion from the ultrasonic motor to the area of interest. Remote actuation can present additional limitations such as, backlash, friction and joint flexibility.

Hydraulic actuators use pressurized hydraulic fluid, such as oil or saline in medical environments (Kim et al., 2002). A hydraulic actuator includes a hydraulic cylinder, a piston with sliding rings and seals, valves, and a supply pressure. Hydraulic actuators can transfer large forces, but the leakage of fluid is an issue in a medical environment. Air bubbles in the fluid could create control problems. Hydraulic actuators are recommended for applications that require high position accuracy, or slow and smooth movements.

Pneumatic actuators have similar construction to hydraulic actuators. Howerver, they use compressed air instead of liquid. Without the threat of the liquid leakage, they are cleaner and are more desirable for a medical environment. Pneumatic actuators can be operated at higher speeds than hydraulic actuators, but due to compressibility of air, they usually provide limited stiffness. Pneumatic actuators are suitable for relatively low-force applications, such as actuating a robot in soft-tissue intervention.

Both hydraulic and pneumatic cylinders made of non-magnetic material can be safely placed close to the imaging areas and will not cause image artifacts. The control valve and control unit should be placed outside of MRI room, or at least outside of the 5 Gauss line.

Pneumatic actuators recently have been used in MRI compatible robotic systems for realtime imaging guided intervention (Hempel et al., 2003, Fischer et al., 2008b, Li et al., 2008). Because of the inter-relationship between temperature, pressure and volume of air, the dynamic control of the pneumatic actuator is challenging. A new concept of pneumatic actuator-PneuStep was presented by Stoianovici and colleagues (Stoianovici et al., 2007b). PneuStep uses a stepper motor principle to achieve precise motion on the order of $0.050 \mathrm{~mm}$.

\subsection{Sensors}

Position and/or force sensors are used to provide feedback for close-loop control of medical robotic devices to provide a safe and accurate operation. Sometimes redundant sensors are used to guarantee the safety in medical intervention systems.

Optical signals do not interfere with magnetic field. Optical sensors have been developed and used for sensing position (Fischer et al., 2008b, Hempel et al., 2003, Hata et al., 2008) and force (Harja et al., 2007, Takahashi et al., 2003) in an MRI environment. A commercially available optical encoder from US digital (Vancouver, Washington, USA) uses transmissive strips for positioning the motion. The reading heads have small electronics to convert the optical signal into an electronic signal. The optical encoder functions well and does not cause image artifacts even if it is placed $5 \mathrm{~cm}$ from the imaging areas (Fischer et al., 2006). Innomedic (Herxheim, Germany) has developed both rotary and linear optical encoders. These magneto-translucent fiber-optical incremental encoders are made of glass. Optical fibers transfer the signals to optoelectronic conversion circuits remotely located. These encoders do not cause image artifacts even if they are placed at the isocenter of the magnet. 
(a)

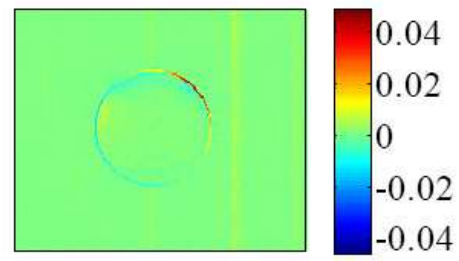

(c)

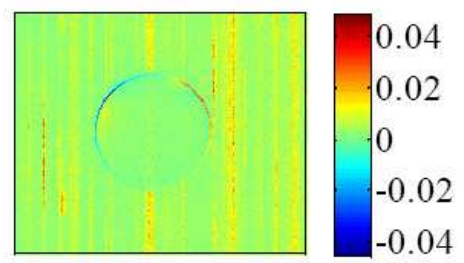

(b)

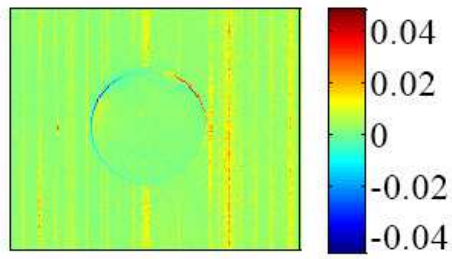

(d)

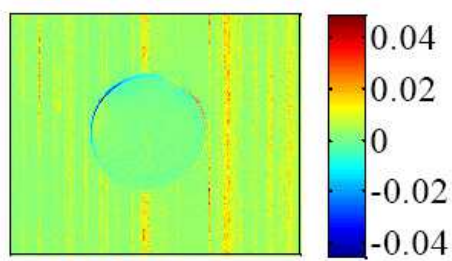

Fig. 1. Mean percentage difference between images under different conditions and baseline image (Phantom only). (a) with robot only (b) with robot inside bore and sensor at $1.0 \mathrm{~m}$ (c) sensor moved to $0.5 \mathrm{~m}$ and (d) sensor moved to $0.25 \mathrm{~m}$

Another type of important optical sensor is for measuring force in an MRI environment. These optical sensors (Harja et al., 2007, Takahashi et al., 2003, Tada \& Kanade, 2005) are used in MRI-compatible robots developed as haptic devices for neuroscience and diagnostics. But they use some electronics which may not function during imaging. Our group modified a commercially available "3D SpaceNavigator" (3dconnexion, Fremont, CA) to create a 6 degrees-of-freedom (DoF) user input sensor which can be placed close to an MRI bore (Kapoor et al., 2009). A "3D SpaceNavigator" optical signal is converted to an electronic signal via a signal PC board, which connects to a USB interface board. After carefully rewiring and shielding, this ergonomic force sensor functions well when it is brought up to $50 \mathrm{~cm}$ of the bore. Figure 1 shows the percentage difference between images under different conditions with the baseline image.

Force sensing can also be achieved by using hydraulics/pneumatics (Liu et al., 2000, Yu et al., 2007). In their work, Gassert and colleagues (Gassert et al., 2006) presented an MR compatible 1-DoF force sensor based on transmission of hydrostatic force and motion which is located outside the MRI room, where traditional electronics can then be used. Due to friction losses and compressibility, there is a significant dead zone in the force sensed output. However, the advantage of using their technique is that it allows use of the device during imaging. 


\section{Mechanical Designs}

Scanners with open configuration present a major advantage regarding the accessibility to the target area. Double-donut MRI scanner offers a $50 \mathrm{~cm}$ vertical gap between two facing superconductive magnets. This gap can accommodate a physician and interventional robots. Another type of open scanner with vertical arrangement of magnet poles at $40-45 \mathrm{~cm}$ distance, also offers good accessibility to the patient. Open-configuration MRI systems with low magnetic fields, in the range of 0.02-0.7 Tesla, result in a lower available signal and slower speeds of image acquisition. The most popular MRI scanners at this time are based on a single superconductive magnet with a horizontal bore. This type of scanner offers better quality imaging with real-time acquisition speeds. The major drawback of these scanners for interventions is the limited space inside the bore, which typically is $60 \mathrm{~cm}$ in diameter and $200 \mathrm{~cm}$ in length. A new generation of scanners (Siemens Espree) with short bore $(120 \mathrm{~cm})$ and increased diameter $(70 \mathrm{~cm})$ make intervention access and interventional robot assistance within the magnet quite feasible.

MRI scanner configuration, the target organ, and the intervention procedure greatly impact the mechanical designs of MRI robots. MRI compatible robots for percutaneous biopsy, drug injection or radiotherapy seed implantation are currently an active research area. We discuss the mechanism of some of these robots and focus on a robotic valve delivery module for beating heart transapical valve replacement in this section.

Chinzei and colleagues presented a robotic system for assisting a physician who stands in the limited space between the two magnets of a double-donut MRI scanner (Chinzei et al., 2000). The base of the robot is mounted above the physician's head in the open MRI magnet and two rigid arms reach down into the surgical field. The robotic device consists of five linear motion stages arranged to form a 2-DoF orienting mechanism, which is attached to a 3-DoF Cartesian positioning mechanism. The ends of the arms are linked to form a tool holder. Recently, Hata and colleagues developed a semi-active needle-guiding manipulator for microwave thermotherapy of liver tumors in a double-donut open MRI (Hata et al., 2008). The manipulator is installed on the side of the patient table at the gap between the magnets. It consists of a 3-DoF Cartesian stage and an encoded passive remote center of motion configuration. The synergistic control keeps the virtual remote center of motion at the pre-planned target using encoder outputs from the needle holder as input to the motorized base stage.

Koseki and colleagues developed a robotic system for endoscope manipulation working inside a vertical-magnetic-field open configuration MRI scanner (Koseki et al., 2002). This 4DoF robot takes advantage of the available horizontal space between the vertical poles of such scanners. They adopted a 5-bar linkage mechanism for 2-DoF translational motions. The planar parallel mechanism reduces the size and enables the actuators to be distant from imaging areas. Tajima and colleagues (Tajima et al., 2004) introduced a master-slave MR robotic system for intraoperative diagnosis and minimally invasive surgery inside an open vertical MRI scanner. Each of the slave manipulators has three translational DoF that are located on its base and three rotational DoF on its arm component. The ultrasonic actuators and sensors were located a meter away from the imaging areas. A mechanism consisting of a pair of differential gears and wires was implemented to transmit movement to the tool tip. Masamune et al. first designed a MRI-compatible manipulator for stereotactic needle therapy (Masamenu et al., 1995) in a conventional closed MRI. The device was designed to 
be mounted on the MRI table and above the head of the subject. It has a stereotactic frame with 6-DoF. The isocentric structure avoids collision with the patient.

There are several reports on MRI compatible manipulators and/or robots for prostate biopsy and brachytherapy. These compact devices are mounted on the MRI table and below the torso. Krieger and colleagues developed a 3-DoF manipulator for MRI guided transrectal prostate (Krieger et al., 2005). The manipulator is equipped with active fiducial tracking to encode the position of the needle path and is remotely manually actuated by the physician from outside of the MRI scanner. The device is comprised of a rectal sheath, which is placed adjacent to the prostate in the rectum of the patient and a needle guide, containing a curved needle channel. Stoianovici and colleagues developed a 5-DoF MrBot actuated by a new type of pneumatic stepper motor for brachytherapy seed placement (Stoianovici et al., 2007a). The MrBot robot is constructed in the form of a platform supported by articulated linear actuators in a 5-DoF parallel link structure. The patient is in the decubitus position and seeds are placed in the prostate transperineally. Fischer and colleagues described a 6DoF robot assistant system for transperineal prostate needle placement (Fischer et al., 2008b). The mechanism consists of two decoupled planar motions. Motion in the vertical plane is achieved using a modified version of a scissor lift mechanism that is traditionally used for parallel plane motion, whereas motion in the horizontal plane is accomplished by coupling two straight line motion mechanisms generally referred to as Scott-Russell mechanisms.

For accessing the organs located in the middle of the torso inside a closed MRI scanner, long flat arm like structure are usually used. Kaiser and colleagues presented ROBITOM, the first MR-compatible robotic system dedicated to MR-guided breast biopsies (Kaiser et al., 2000). The entire assembly is a box-like structure that is anchored on top of the standard MRI scanner table. The patient lies prone on the elevated table with the breasts placed through two openings. Larson and colleagues developed a similar breast-dedicated MR-compatible robotic system (Larson et al., 2004) with multiple degrees of freedom and remote control. Their device is remotely actuated with ultrasonic motors and a motion transmission system consisting of shafts and universal joints. Innomotion (Innomedic GmbH, Herxheim, Germany) is a commercially available MR-compatible interventional robotic system, original developed for precise needle insertion for MR-guided therapy of spinal diseases. An arch is mounted to the MRI patient bed with specially made rails. An arm sitting on the arch takes advantage of the clearance between the patient and the bore. The robot has 5-DoF with a remote center of motion structure to place and orient an interventional tool.

\subsection{Robotic module for transapical aortic valve replacement}

Our group has developed a robotic system for transapical aortic valve replacement under real-time MRI guidance ( $\mathrm{Li}$ et al., 2008). The robotic component consists of a 5-DoF robotic arm and a 3-DoF valve delivery module. The 5-DoF Innomotion robotic arm is employed as the 3-DoF valve delivery module holder. The compact valve delivery module is designed for manipulating and placing the prosthesis into a beating heart, while in the MRI scanner. The valve delivery module consists of a sterilizable valve delivery unit and an active mechanism that provides the essential manipulation of the delivery device for valve placement. 


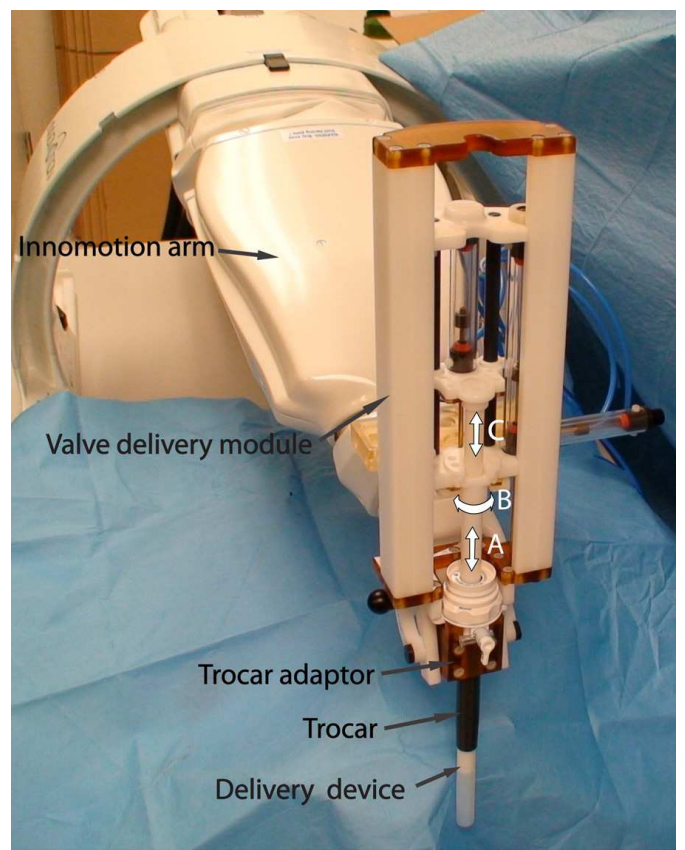

Fig. 2. Picture of the robotic system for MRI guided transapical aortic valve replacement. It shows an Innomotion arm for the positioning module and the prototype of the new developed 3-DoF valve delivery module affixed on the robotic arm.

The valve delivery unit includes a delivery device, a trocar, and a trocar adaptor. The delivery device consists of a straight plastic rod, outside of which is a sheath protecting the prosthetic valve before it is deployed. The robotic active mechanism comprises one rotational joint (B) and two linear joints: the translation joint (A) and the insertion joint (C) (fig 2). The operations of linear and rotational joints are independent.

The translation joint provides linear displacement of the delivery device along its axis. This joint is directly actuated by two parallel linear pneumatic actuators. The two-actuator structure guarantees balanced motion of a delivery device. The bodies of two linear actuators, together with a base plate, a top plate and two inner rails, form a rigid sliding frame. The sliding frame can slide on two frame rails. These two frame rails, along with a connecting plate (for mounting on a positioning arm) and a top plate form a rigid base frame. The position of the motion is sensed by a linear optical encoder that is attached to a frame rail of the rigid frame. This travel range of this joint is $90 \mathrm{~mm}$.

The rotational joint rotates the delivery device around its axis. This changes the orientation of the prosthesis relative to coronary ostia before it is deployed. It is actuated by a linear pneumatic actuator attached on the base plate of the sliding frame. The linear movement is transmitted to the rotational movement by a rack-gear transmission. The rotation angle is sensed by a linear optical encoder. A sliding base rack actuated by the pneumatic actuator transmits linear movement to the gears of the rotation axis. The travel range of this joint is \pm 60 degree. 
The other linear joint is an insertion joint. This linear movement advances the prosthetic valve out of the delivery device. One central sliding stage with a semicircular groove actuated by a linear actuator can slide on the inner rails of the rigid sliding frame. The whole travel length of the insertion is $45 \mathrm{~mm}$, which guarantees the prosthesis be driven out from the sheath. This linear movement is also encoded with an optical linear encoder.

The prototype of the valve delivery module is shown in Figure 2. All parts of the prototype delivery module are made from non-conductive plastic materials. Pneumatic actuators and optical sensors are used for operating and positioning each joint. The robotic module allows precise and repeatable positioning of a bioprosthetic valve in the correct location. The maximum measured force load of the translation stage, under 75 psi operation air pressure, was $34 \mathrm{~N}$; and the maximum torque of the rotation stage was $0.4 \mathrm{Nm}$. With operational velocity of $10 \mathrm{~mm} / \mathrm{sec}$, the accuracy of translation stage was found to be $0.19 \pm 0.14 \mathrm{~mm}$. With operational velocity of $5 \mathrm{deg} / \mathrm{sec}$, the accuracy of the rotation stage was found to be $0.46 \pm 0.27 \mathrm{deg}$. With a load of $10 \mathrm{~N}$ (a reasonable force if we consider the friction and reaction force from a real case), the module moved smoothly and stably and the accuracy was in the same range. The presence and motion of the robotic module inside the scanner was found to have no noticeable disturbance to the image. The observed signal-noise-ratio (SNR) loss was $6.1 \%$ to $6.5 \%$ for the valve delivery module placed in the bore and in motion respectively.

\section{Control Strategies}

Improving precision and accuracy, while maintaining compatibility and safety with MRI are the prime concerns for most MRI compatible robotic systems. Point-to-point position motion is the basic control mode for these robots. However, a shared control between a user and a MRI compatible robot makes it a more intuitive instrument especially during the setup phases of interventions. We describe the needs and method to implement the hands-on coorperative control mode on a MR compatible robot in this section.

Planning a minimally invasive intervention on living tissue is not a trivial task. The organs move around and thus the pre-planned motion based on pre-operative images alone is not sufficient.

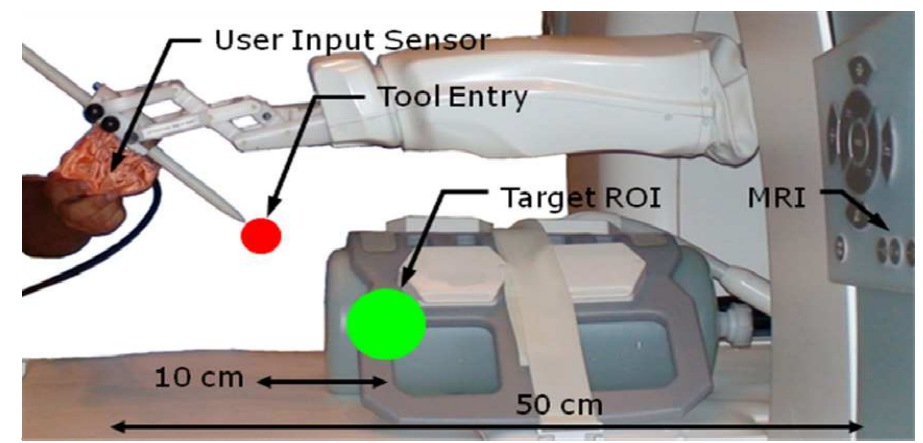

Fig. 3. Hands-on cooperative control

Most of the contemporary systems make use of intra-operative images and a graphical user interface to update the planned motions. It may be not necessary and sometimes impractical 
to have an image guided interface for the robot during procedure setup. In the robotic assisted intervention of beating heart transapical valve replacement ( $\mathrm{Li}$ et al., 2008), before the prosthetic valve delivery process can be started, the surgeon must perform preparatory procedures of placing the trocar into the apex of the heart. Thereafter, the surgeon loads the delivery device with the prosthetic valve and inserts the delivery device into the trocar. Using imaging to guide the placement at this stage was unnecessary and impractical because of the large motion and variability in the localization of the trocar. Typically, the trocar port is about $10-15 \mathrm{~cm}$ from imaging center, thus requiring a very large image acquisition volume. Breathing motion of up to $20 \mathrm{~mm}$ also causes localization and registration errors. Moreover, adjustments may also be required to the entry point after a preliminary scan of the delivery device is acquired.

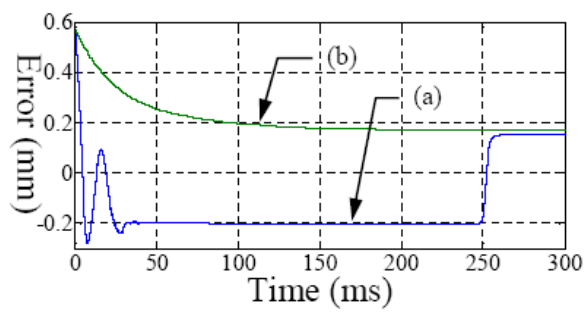

Fig. 4. Position error in the PIV controller for one of the robot axis under two different modes. (a) Gains optimized for point-to-point mode (b) Gains for hands-on mode.

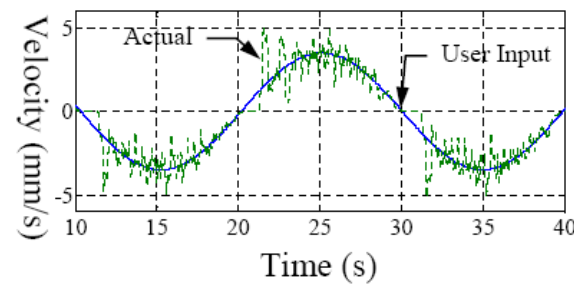

(a)

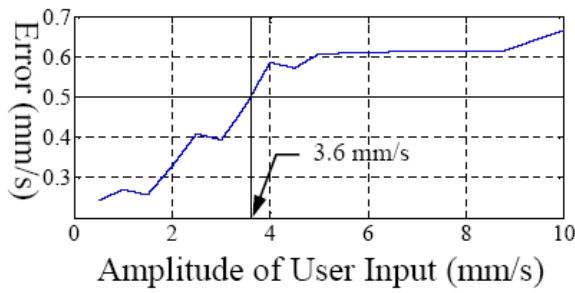

(b)

Fig. 5. (a) User input with amplitude of $3.5 \mathrm{~mm} / \mathrm{s}$ and corresponding actual Cartesian velocity (b) Average Cartesian velocity error over time (2 cycles) with respect to amplitude of user input.

Figure 3 shows the setup of the Innomotion robotic arm and user input sensor in the MRI room. We describe the method to implement the hands-on coorperative control mode on a modified pneumatically actuated Innomotion robotic arm (Innomedic, Herxheim, Germany). The actuators of the Innomotion robot are controlled by an off-the-shelf controller (Motion Engineering Inc/Danaher Motion, Santa Barbara, CA). A PIV (proportional position loop integral and proportional velocity) controller runs on the proprietary DSP based hardware. 


\subsection{Low level control}

One important difference between hands-on control mode and other modes is that the later prefers zero or low steady state errors. Hands-on mode has a user in the control loop who can tolerate a fair amount of steady-state error by adjusting his/her input to achieve the desired result. This mode demands a fast and smooth response without oscillations during the transient as well as steady state. Low level PIV gains should satisfy this requirement. It is straight forward to obtain such a response on traditional motor driven robots. However, for a pneumatic robot a compromise between smoothness, speed and error has to be made. To achieve this behavior, we applied a sine wave as user input (velocity) with varying amplitudes. Iteratively, we adjusted the PIV gains and the maximum amplitude of user input that would produce reasonable error, till we saw no further improvement. In figure 4, the fast oscillatory response for point-to-point gains is shown along with a smoother, reasonably fast response for hands-on mode. Figure 5(a) shows a typical profile for a user input with amplitude of $3.5 \mathrm{~mm} / \mathrm{sec}$ and the actual Cartesian velocity measured using robot encoders. The error is defined as the difference between the user input and the actual Cartesian velocity at that instant. The average error is computed by averaging the error over the number of samples collected during two cycles of user input. Figure 5(b) shows this value for different amplitudes of user input. Our current system can tolerate a maximum user input speed of $3.6 \mathrm{~mm} / \mathrm{sec}$ and $3.6 \mathrm{deg} / \mathrm{sec}$, if the required error is to be kept below $0.5 \mathrm{~mm} / \mathrm{sec}$ and $0.5 \mathrm{deg} / \mathrm{sec}$.

\subsection{High level admittance control}

Depending on how the inertias and friction forces compare with the forces applied by the environment to the robot, it is possible to classify robotic devices into two categories: impedance-type and admittance-type. More importantly, it is the control scheme that distinguishes the two types. The impedance-type robots act as a force/torque source, where the controller outputs a force based on desired input positions (and their derivatives). In admittance-type robots, the controller outputs a desired position (and its derivatives) based on user inputs such as force measurement or joystick motion. Innomotion robot is practically "non-back-drivable", i.e., it requires significant effort to overcome internal friction to maneuver it. Thus, it is more suited for an admittance control scheme such as those explored with the Johns Hopkins University Steady-Hand Robot (Taylor et al., 1999). In their work, force sensors could be used to obtain user input. However, MRI compatibility poses certain challenges in obtaining a 6-DoF user input. The outline of our hands-on controller is as follows:

1) Switch the low-level PIV gains to ones optimized for hands-on mode.

2) Obtain incremental motion desired by the user, that is $\dot{x}=K_{c} \cdot f$, where $\dot{x}$ is desired incremental motion, $f \in R^{6}$ is user input and $K_{c}$ is a scaling matrix.

3) Compute current joint state, $q$, from current actuator values, $a$, and incremental motion in the actuators, $\Delta a$.

4) A constrained least squares problem is solved for the optimal incremental joint motion, $\Delta q^{*}$ by the high-level controller. The least-squares (LS) problem has an objective function describing the desired outcome. It includes constraints that consider joint limits, and importantly velocity limits. 


$$
\begin{aligned}
& \Delta q^{*}=\arg \min _{\Delta q}\left\|W_{c} \cdot\left(\Delta x-K_{c} f\right)\right\|+\left\|W_{q} \cdot \Delta q\right\| \\
\text { s.t. } & |q| \leq \dot{q}_{U} \cdot \Delta t \\
& q_{L} \leq q+\Delta q \leq q_{U} \\
& \Delta x=J \cdot \Delta q
\end{aligned}
$$

The incremental joint motion, $\Delta q$, is a variable for LS problem. Matrix $J$ is the Jacobian of the robot. $q_{L}$ and $q_{U}$ are the lower and upper bounds of the joint variables. $\dot{q}_{U}$ is the upper bound of the joint velocities that are obtained as described in earlier section. $\Delta t$ is the small time interval of the high-level control loop. Without any constraints, the above constrained LS problem, which is implemented in the high-level block, is equivalent to resolved rate control. $W_{c}$ and $W_{q}$ are diagonal weight matrices.

5) Numerically integrate the incremental joint motion to arrive at a new set of joint positions. We assume that for each iteration loop, the incremental motions are sufficiently small and $\Delta x / \Delta t=J \cdot \Delta q / \Delta t$ represents a good approximation to the instantaneous kinematics.

6) Compute desired actuator values, $a^{d}$ and desired actuator velocities, $\dot{a}^{d}=\Delta a^{d} / \Delta t$ from $q$ and $\Delta q^{*}$. These are new set points for the low-level controller.

7) Repeat steps 2-6 while in hands-on mode. On exit, switch PIV gains to point-to-point mode values.

Numerical integration and rate control laws such as these are known to be "nonconservative" and may result in positional errors. However, this is not a concern here, since a human-in-the-loop can easily account for any positional error by applying required additional input to reach the desired goal. The desirable behavior here is that the robot continues to follow the user input as best as it can, even in the advent of certain limits, such as joint or velocity extremity.

\subsection{Experiments and Results}

We tested the system functionality, as well as, the qualitative ease of use of the sensor. We also evaluated the hands-on sensor interface with respect to current and alternate interfaces. We used a quintessential peg-in-the-hole task to emulate the clinical scenario that inserts a dexterous tool into a trocar inside a thoracic cavity. Our peg was a cylinder $12.5 \mathrm{~mm}$ in diameter and $140 \mathrm{~mm}$ long, which mimicked the dimension of the delivery device. To design our experiments to be in line with Fitt's law (Zhai, 1995), we used two different hole dimensions $(13.5 \mathrm{~mm}$ and $16.5 \mathrm{~mm})$ for fine and coarse positioning accuracy, respectively. Further, the larger hole had a larger bevel at the entry point. The hole was placed at a known position with respect to the robot and the starting configuration of the robot was chosen at random for each trial. The starting position was chosen using an uniform random distribution from a spherical annular region of radii $15 \sqrt{3}$ to $20 \sqrt{3} \mathrm{~mm}$, and the orientations were picked in the interval of $\pm 15- \pm 20 \mathrm{deg}$. These are clinically relevant distances, because the robot can be easily and quickly positioned to within these regions using available passive adjustments. 
For comparision, we developed a simplified GUI that resembles the commands acceptable to the AESOP, that is, the surgeon could annunciate one of "move"-"left", "right", "up", "down", "front", "back", or "rotate"-"left", "right", "front" and "back" to move the 5-DoF robot in the appropiate direction. The person at console would have to press the corresponding button. The buttons to increase/decrease speed would change the current set speed by $0.5 \mathrm{~mm} / \mathrm{sec}$. In our experimental setup, the person at console could not directly visualize the robot to replicate the clinical setting. The person at console was picked at random from the pool of users to avoid bias.

Each user was asked to perform the task as quickly as possible, on the "start" cue of the person on console and indicate verbally when they completed the task by announcing "done". Comparisons of task times recorded using a stop watch have been shown in figure 6. As seen, hands-on approach is efficient for both the levels of accuracy.

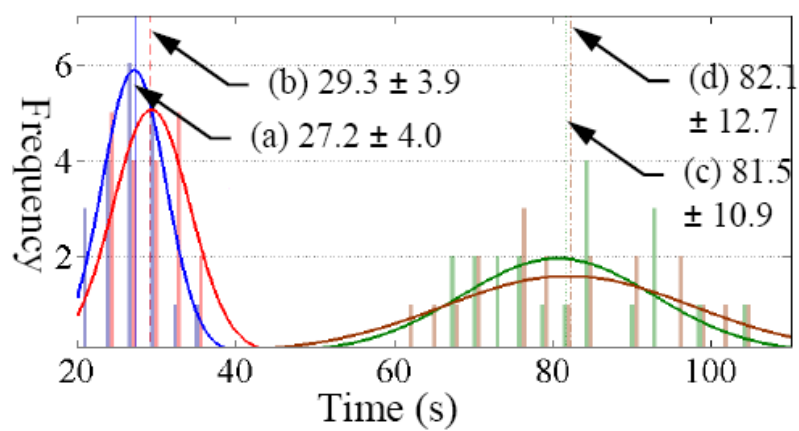

Fig. 6. Histogram of time required to complete the peg-in-hole task $(a, b)$ hands-on and hole size of $16.5 \mathrm{~mm}$ and $13.5 \mathrm{~mm}$, respectively $(\mathrm{c}, \mathrm{d})$ AESOP like interface and hole size of $16.5 \mathrm{~mm}$ and $13.5 \mathrm{~mm}$, respectively. The solid curves are the fitted Gaussian distributions with means and deviations as indicated.

This can be attributed to the ability to perform complex continuous motions involving multiple translational and rotational degrees of freedom at one time. Further, using the GUI interface, the user announcing the commands has to contemplate his/her next moves. As expected, there is an increase in time required with an increase in the difficulty of the task. Though, this difference is not significant when compared with the difference between the two approaches.

\section{Conclusion}

MRI provides excellent visualization of soft tissue, its sub-structure and surrounding tissues. The unique features of MRI, such as oblique image planes, multi-slice imaging, realtime visualization, and freedom from radiation exposure risk, enable MRI to be a critical tool in the guidance of many interventional procedures. Additionally, a mechatronic system can provide more accurate and smooth access to targeting organs in a confined space. The marriage of a MRI and a robot makes the benefit of minimally invasive interventions substantial. 
The high magnetic field and the confined space of MR bore presented many technical challenges. The design of robotic system is dependent of MRI scanner configuration type, medical intervention and target areas. The mechatronic components including actuators, sensors and controllers must be able to work in accurate, stable and robust way in MR environment. Materials used for robotic system should have low magnetic susceptibilities (comparable with air, water or human tissue), low electrical conductibility, adequate mechanical strength and good manufacturing properties. Certain conductive materials can be used, but only for small parts and without loops. If it is necessary, electrical components may be brought into MR environment if their electrical signals are of low frequency and are shielded.

Control strategy and human machine interface for MRI compatible robotic systems for medical interventions need to be studied. A shared control between a user and an MRI compatible robot makes it a more intuitive instrument especially during setup phases of interventions. An image-guided interface would be ideal to plan and manipulate the robotic holder or adjust the interventional tool when the robot is inside the magnet bore. In the engineering of robots for medical applications, detailed analyses of the functions of the entire system, that is, robot, interfaces and application, taken as single entity, are arguably more important than the individual performance of the subsystems (robot, surgeon, interfaces and application, separately). Thus, having a combination of more than one interface such as an image-guided, console guided or hands-on based on application might yield a higher performance from the entire system.

\section{References}

Bock, M.; Volz, S.; Zuhlsdorff, S. \& et al. (2004). MR-guided intravascular procedures: Realtime parameter control and automated slice positioning with active tracking coils. Journal of Magnetic Resonance Imaging. Vol.19, 580-589

Chinzei, K.; Kikinis, R. \& Jolesz, F. (1999). MR Compatibility of Mechatronic Devices: Design Criteria. Proc. of MICCAI' 99 Lecture Notes in Computer Science. Vol.1679/1999, 10201031.

Chinzei, K.; Hata, N.; Jolesz, F.A. \& et al. (2000). MR compatible surgical assist robot: system integration and preliminary feasibility study. Proc. of MICCAI '00 Lecture notes in Computer Science. Vol.1935/2000, 921-930.

Elhawary, H.; Zivanovic, A.; Rea, M \& et al. (2007). A MR compatible mechatronic system to facilitate magic angle experiments in vivo. Proc. of MICCAI '07 Lecture notes in Computer Science. Vol.4792/2007, 604-611

Fischer, G.S.; Iordachita, I.; DiMaio, S.P. \& et al. (2006). Design of a Robot for Transperineal Prostate Needle Placement in an MRI Scanner, Proc. of IEEE International Conference on Mechatronics. pp 592-597, Budapest, Hungary.

Fischer, G.S.; Krieger, A.; Iordachita, I. \& et al. (2008a). MRI Compatibility of Robot Actuation Techniques - A Comparative Study. Proc. of MICCAI '08 Lecture Notes in Computer Science. Vol.5242/2008, 509-517

Fischer, G.S.; Iordachita, I.; Csoma, C. \& et al. (2008b). MRI-Compatible Pneumatic Robot for Transperineal Prostate Needle Placement. IEEE/ASME Transactions on Mechatronics. Vol.12(3), 295-305. 
Gasset, R.; Moser, R.; Burdet, E. \& et al. (2006). MRI/fMRI-compatible robotic system with force feedback for interaction with human motion. IEEE/ASME Transactions on Mechatronics. Vol. 11(2), 216-224

Guttman, M.A.; Kellman, P.; Dick, A.J. \& et al. (2003). Real-time accelerated interactive MRI with adaptive TSENSE and UNFOLD. Magnetic Resonance in Medicine. Vol.50, 315321

Harja, J.; Tikkanen, J.; Sorvoja, H. \& et al. (2007). Magnetic resonance imaging-compatible, three-degrees-of-freedom joystick for surgical robot. International Journal of Medical Robotics and Computer Assisted Surgery. Vol.3(4), 365-371

Hata, N.; Hashimoto, R.; Tokuda, J. \& et al. (2005). Needle guiding robot for MR-guided microwave thermotherapy of liver tumor using motorized remote-center-of-motion constraint. Proc. of IEEE International Conference on Robotics \& Automation. pp. 16521656, Barcelona, Spain.

Hempel, E.; Fischer, H.; Gumb, L. \& et al. (2003). An MRI-compatible surgical robot for precise radiological interventions. Computer Aided Surgery. Vol.8(4), 180-191

Henk, C.B.; Higgins, C.B.; \& Saeed, M. (2005). Endovascular interventional MRI. Journal of Magnetic Resonance Imaging. Vol.22, 451-460.

Horvath, K.A.; Guttman, M.; Li, M. \& et al. (2007) Beating heart aortic valve replacement using real-time MRI guidance. Innovations. Vol.2, 51-55

Jolesz, F.A. (1998). Interventional and intraoperative MRI: a general overview of the field. Journal of Magnetic Resonance Imaging. Vol.8, 3-7

Kaiser, W.A.; Fischer, H.; Vagner, J. \& et al. (2000). Robotic system for biopsy and therapy of breast lesions in a high-field whole-body magnetic resonance tomography unit. Investigative Radiology. Vol.35, 513-519

Kapoor, A.; Wood, B.; Mazilu, D. \& et al. (2009). MR-compatible Hands-on Cooperative Control of a Pneumatically actuated robot. Proc. of IEEE International Conference on Robotics \& Automation. pp. 2681-2686, Kobe, Japan.

Kim, D.; Kobayashi, E.; Dohi, T. \& et al. (2002). A new, compact MR-compatible surgical manipulator for minimally invasive liver surgery. Proc. of MICCAI '02 Lecture Notes in Computer Science. Vol.2488/2002, 164-169

Koseki, Y.; Washio, T.; Chinzei, K. \& et al. (2002). Endoscope manipulator for trans-nasal neurosurgery, optimized for and compatible to vertical field open MRI. Proc. of MICCAI '02 Lecture Notes in Computer Science. Vol.2488/2002, 114-21.

Krieger, A.; Susil, R.C.; Menard, C. \& et al. (2005). Design of a novel MRI compatible manipulator for image guided prostate interventions. IEEE Transactions on Biomedical Engineering. Vol. 52, 306-313

Kuehne, T.; Saeed, M.; Higgins, C.B. \& et al. (2003). Endovascular stents in pulmonary valve and artery in swine: feasibility study of MR imaging-guided deployment and postinterventional assessment. Radiology. Vol.226, 475-481.

Larson, B.T.; Erdman, A.G.; Tsekos, N.V. \& et al. (2004). Design of an MRI-compatible robotic stereotactic device for minimally invasive interventions in the breast. Journal of Biomechanical Engineering. Vol.126, 458-65

Lederman, R.J. (2005). Cardiovascular interventional magnetic resonance imaging. Circulation. Vol.112, 3009-3017. 
Li, M.; Mazilu, D. \& Horvath, K. (2008). Robotic system for transapical aortic valve replacement with MRI guidance. Proc. of MICCAI '08 Lecture Notes in Computer Science. Vol.5242/2008, 476-484

Liu, J.Z.; Dai, T.H.; Elster. T.H. \& et al. (2000). Simultaneous measurement of human joint force, surface electromyograms, and functional MRI-measured brain activation. Journals of Neuroscience Methods. Vol.101(1), 49-57

McVeigh, E.R.; Guttman, M.A.; Lederman, R.J. \& et al. (2006). Real-Time Interactive MRIGuided Cardiac Surgery: Aortic Valve Replacement Using a Direct Apical Approach. Magnetic Resonance in Medicine. Vol.56, 958-964.

Melzer, A. \& Seibel, R. (1999). MRI-guided treatment of degenerative spinal diseases. Minimally Invasive Therapy \& Allied Technologies. Vol.8(5), 327-335

Nayak, K.S.; Cunningham, C.H.; Santos, J.M. \& et al. (2004). Real-time cardiac MRI at 3 Tesla. Magnetic Resonance in Medicine. Vol.51, 655-660

Masamune, K.; Kobayashi, E.; Masutani, Y. \& et al. (1995). Development of an MRIcompatible needle insertion manipulator for stereotactic neurosurgery. Computer Adied Surgery. Vol.1, 242-248

Raval, A.N.; Karmarkar, P.V.; Guttman, M.A. \& et al. (2006). Real-time magnetic resonance imaging-guided endovascular recanalization of chronic total arterial occlusion in a swine model. Circulation. Vol.113, 1101-1107.

Schenck, J.F. (1998). MR safety at high magnetic fields. Magnetic Resonance Imaging Clinics of North America. Vol.6, 715-30

Schenck, J.F. (2000). Safety of strong, static magnetic fields. Journal of Magnetic Resonance Imaging. Vol.12, 2-19

Stoianovici, D.; Song, D.; Petrisor, D. \& et al. (2007a). "MRI Stealth" robot for prostate interventions. Minimally Invasive Therapy \& Allied Technologies. Vol.16, 241-248

Stoianovici, D.; Patriciu, D; Petrisor, D. \& et al. (2007b). A new type of motor: Pneumatic step motor. IEEE/ASME Transactions on Mechatronics. Vol.12(1), 98-106

Tada, M. \& Kanade, T. (2005). Design of an MR-compatible three-axis force sensor. Proc. of IEEE/RSJ International Conference on Intelligent Robots and Systems. pp. 3505-3510, Edmonton, Canada.

Tajima, F.; Kishi, K.; Nishizawa, K. \& et al. (2004). A Prototype Master-Slave System Consisting of Two MR-Compatible Manipulators with Interchangeable Surgical Tools Part of a Unified Support System for Diagnosis and Treatment. Proc. of IEEE International Conference on Robotics $\mathcal{E}$ Automation, pp. 2505-2510, New Orleans, USA.

Takahashi, N.; Tada, M.; Ueda, J. \& et al. (2003). An optical 6-axis force sensor for brain function analysis using fMRI. Proc. of IEEE Sensors, pp. 253-258, Toronto, Canada.

Taylor, R.H.; Jensen P.; Whitcomb, L.L. \& et al. (1999). A Steady-Hand robotic system for microsurgical augmentation. International Journal of Robotics Research. Vol.18(12), 1201-1210

Yu, N.; Murr, W; Blickenstorfer, A. \& et al. (2007). An fMRI compatible haptic interface with pneumatic actuation. Proc. of IEEE International Conference on Rehabilitation Robotics, pp. 714-720, Noordwijk, Netherlands

Zhai, S. (1995). Human Performance in Six Degree of Freedom Input Control. Ph.D. dissertation, University of Toronto 


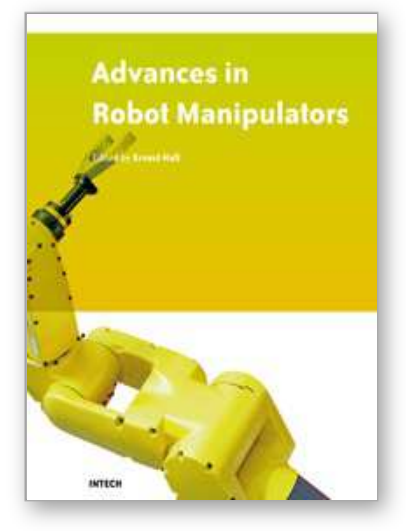

\author{
Advances in Robot Manipulators \\ Edited by Ernest Hall
}

ISBN 978-953-307-070-4

Hard cover, 678 pages

Publisher InTech

Published online 01, April, 2010

Published in print edition April, 2010

The purpose of this volume is to encourage and inspire the continual invention of robot manipulators for science and the good of humanity. The concepts of artificial intelligence combined with the engineering and technology of feedback control, have great potential for new, useful and exciting machines. The concept of eclecticism for the design, development, simulation and implementation of a real time controller for an intelligent, vision guided robots is now being explored. The dream of an eclectic perceptual, creative controller that can select its own tasks and perform autonomous operations with reliability and dependability is starting to evolve. We have not yet reached this stage but a careful study of the contents will start one on the exciting journey that could lead to many inventions and successful solutions.

\title{
How to reference
}

In order to correctly reference this scholarly work, feel free to copy and paste the following:

Ming Li, Dumitru Mazilu, Ankur Kapoor and Keith A. Horvath (2010). MRI Compatible Robot Systems for Medical Intervention, Advances in Robot Manipulators, Ernest Hall (Ed.), ISBN: 978-953-307-070-4, InTech, Available from: http://www.intechopen.com/books/advances-in-robot-manipulators/mri-compatible-robotsystems-for-medical-intervention

\section{INTECH}

open science | open minds

\section{InTech Europe}

University Campus STeP Ri

Slavka Krautzeka 83/A

51000 Rijeka, Croatia

Phone: +385 (51) 770447

Fax: +385 (51) 686166

www.intechopen.com

\section{InTech China}

Unit 405, Office Block, Hotel Equatorial Shanghai

No.65, Yan An Road (West), Shanghai, 200040, China

中国上海市延安西路65号上海国际贵都大饭店办公楼 405 单元

Phone: +86-21-62489820

Fax: +86-21-62489821 
(C) 2010 The Author(s). Licensee IntechOpen. This chapter is distributed under the terms of the Creative Commons Attribution-NonCommercialShareAlike-3.0 License, which permits use, distribution and reproduction for non-commercial purposes, provided the original is properly cited and derivative works building on this content are distributed under the same license. 